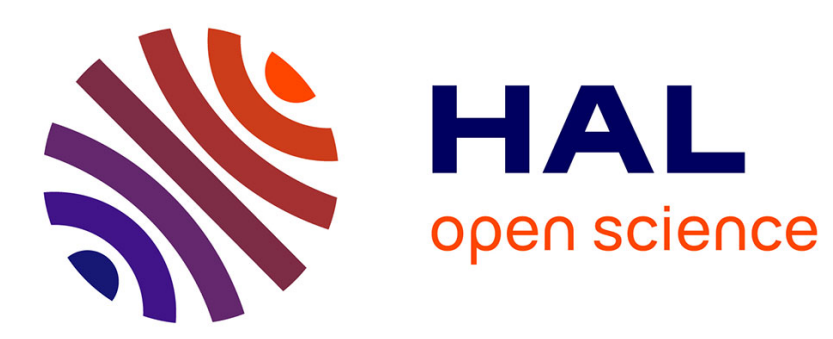

\title{
Reversible Magnetization of Ferrite-Garnet Film by Polarized Light
}

\author{
V. Sohatsky, V. V. Kovalenko
}

\section{To cite this version:}

V. Sohatsky, V. V. Kovalenko. Reversible Magnetization of Ferrite-Garnet Film by Polarized Light. Journal de Physique IV Proceedings, 1997, 07 (C1), pp.C1-699-C1-702. 10.1051/jp4:19971285 jpa00254998

\section{HAL Id: jpa-00254998 https://hal.science/jpa-00254998}

Submitted on 1 Jan 1997

HAL is a multi-disciplinary open access archive for the deposit and dissemination of scientific research documents, whether they are published or not. The documents may come from teaching and research institutions in France or abroad, or from public or private research centers.
L'archive ouverte pluridisciplinaire HAL, est destinée au dépôt et à la diffusion de documents scientifiques de niveau recherche, publiés ou non, émanant des établissements d'enseignement et de recherche français ou étrangers, des laboratoires publics ou privés. 


\title{
Reversible Magnetization of Ferrite-Garnet Film by Polarized Light
}

\author{
V. Sohatsky and V. Kovalenko \\ Department of Radiophysics, Kiev University, Vladimirskaya 64, 252017, Kiev, Ukraine
}

\begin{abstract}
The effect of low-powered polarized light on magnetic characteristics of ferrimagnetic garnet films has been studied at room temperatures by means of magneto-optical Faraday effect. A local magnetization of the film, occurred under the illumination, was carried out in the direction determined by orientation of polarization vector. The effect, accompanied by the domain structure reconstruction, caused also changes of the mechanical strain and dichroism. The spectral, polarization, amplitude and temperature dependences were investigated in order to explain the-origin of such a photomagnetic effect. Peculiarities of the effect and their correlation with parameters of the films and illuminated light are discussed in terms of the developed early models, based on light induced recharging of the polarization-sensitive anisotropic centres. The additional magnetostriction mechanism is assumed to be responsible for displaying of the observed phenomena.
\end{abstract}

\section{INTRODUCTION}

\subsection{Low-temperature effects}

Polarization-dependent effect of light on magnetic, mechanical and optical characteristics of monocrystalline yttrium iron garnet doped by silicon (YIG:Si) was observed previously [1] at temperatures below $140 \mathrm{~K}$ and in bulk samples mainly. This effect of polarized light can be reversible, so the primary state is restored by repeated illumination of the sample by the orthogonal polarized light. The irreversible influence of non-polarized light on ferrimagnetics was revealed at temperatures up to $200 \mathrm{~K}$ and even higher. Some authors [2] reported a weak influence of non-polarized light on magnetic characteristics (permeability, anisotropy) of ferrite- garnet films at also cryogen temperatures. In case of non-polarized light effect, the primary state of the sample can be restored only by its heating up to room temperature.

These results caused the earliest interest concerning so-called photomagnetic effect (PME), that was connected with the hopes for its application in magneto-optics. That hopes couldn't come true, in particular, because of the low temperatures. A further interest was maintained due to the interesting physical phenomenon of light interaction with the rare earth-transition metal crystals, that have electric and magnetic subsystems, strongly affecting one another. The electron transitions between the ions under illumination cause changes of the ions distribution on inequivalent sites of the crystal lattice, that consequently can change some characteristics of the substance. Although it is certain that the induced $\mathrm{Fe} 2+$ ions are responsible for the spin-reorientational transitions in iron garnets, the mechanism of the PME is not well understood. The problem of concrete identification of photoactive centres and their basic and excited states are not solved until now.

\subsection{Room-temperature effects}

A new progress in investigations of the PME has been done recently, when the influence of polarized light on remagnetization of Co-doped garnet film under the external alternative or permanent magnetic field had been observed [3]. This report allows to estimate some necessary parameters of the films desirable for displaying of the "hightemperature" PME. The obvious condition was a small value of the energy of remagnetization. As the nearly phase instability state can be easily attained in the films with a planar anisotropy, we have mainly investigated such films searching for the effect.

As a result, the observation of room-temperature polarization- dependent PME [4] became possible in ferritegamet films, when they were illuminated by the linearly polarized light of $2 \mathrm{~mW}$ He-Ne laser. In spite of thermal 
disordering, the domain structure (DS) was being reconstructed only under the illumination. But manifestation of the described effect is not restricted to reconstruction of the DS. At the same time magnetization, mechanical strain and optical dichroism also have been changed. As the DS reconstruction is the most expressive displaying of the PME, it gives the main information about its regularities.

\section{EXPERIMENTAL}

By means of polarization microscope we were able to investigate some static and dynamic characteristics of magnetization reversal processes in ferrite-garnet films. The typical selected films of composition (YBi) $3 \mathrm{Fe}_{5} \mathrm{O}_{12}$ doped with Tm,Lu of above 5 micron thickness were grown by liquid phase epitaxy on (100)-plane of gallium gadolinium garnet substrate. An "easy plane" anisotropy has been obtained by varying the quantity of impurities.

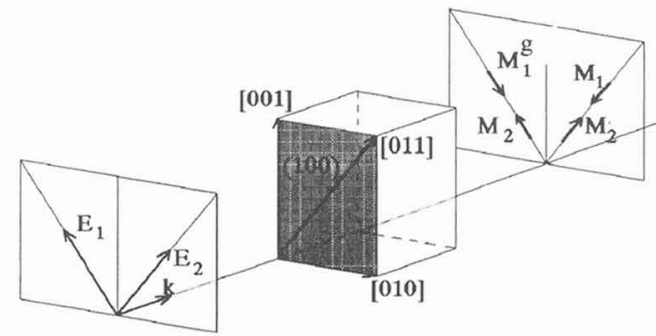

Figure 1. Spatial orientation of the light polarization, crystallography axes and vectors of magnetization.

The experimental installation, used Faraday effect, was consisted of polarized light sources (lasers with $0.488,0.53$, 0.63 wavelength, $\mathrm{P}=1-15 \mathrm{~mW}$ or the filament lamp with the colour filters), optical focusing and control systems, ferritegarnet film, photodetector and videorecorder. The additional weak non-destructive read-out laser beam was used as a monitor. It passed through the film, polarizer and was registered by the photodetector. The direction of this beam was slightly inclined to the (100)-plane for measure Faraday component of magnetization. The intensity of the read-out beam was being changed in proportion with the DS reconstruction. At the same time the DS images were vizually controlled and recorded. Simultaneously with magneto-optical registration, the intensity of magnetization was measured by the magnetoresistance probe, set close to the film surface.

\subsection{Domain structure reconstruction and magnetization}

The displaying of the PME was of two main types: i) reorientation of magnetic vectors in illuminated area in the opposite direction accompanied by the local magnetization of the film (Fig.2c); ii) reorientation of magnetic vectors in perpendicular direction of another easy anisotropy axis (Fig.2g) without magnetization.

i) Illumination of the film by the light with polarization vector $\mathbf{E}_{1} \mid[0-11]$ leads to increasing of the domains with $\mathbf{M}_{2}$ I [011] and to correspondent decreasing of the domains with $\mathbf{M}_{1}$ opposite to $\mathbf{M}_{2}$. The directions of magnetization are shown by the arrows on the domains. Switching off the light has keeped the results of photoinduced changes constant except the neglect relaxation part. After irradiation of the same film area by orthogonal polarized light with $\mathbf{E}_{2} \mid$ [ [0 II], one can remagnetize it in the opposite direction. Such a reconstruction carrying out by means of the domain walls displacement is similar to remagnetization under the external magnetic field, however, unlike [3] we did not use the additional magnetic fields for displaying of the effect. In order to decrease the affect of the carth's magnetic field, we oriented the film perpendicular to the lines of magnetic force and keeped it motionless during the experiments.
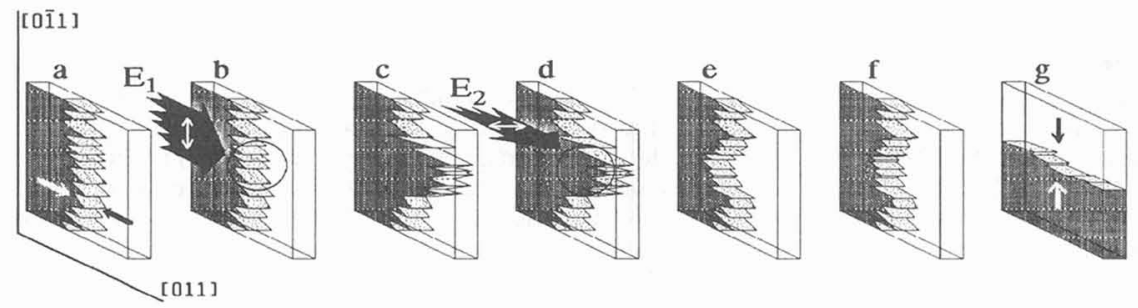

Figure 2. Successive states of the DS in garnet film under illumination.

ii) There were observed another type of the DS reconstruction, when magnetization vectors were reoriented abrupt from one to another orthogonal easy anisotropy axis in (100)-plane (Fig $2 \mathrm{~g}$ ). In this case a small external magnetic field, directed

between the [0-11] and [011] axes was used. It was stayed invariable while the direction of the light polarization had switched between the easy anisotropy axes. The field did not influence the DS and the film is stayed demagnetized in this case. Owing to such external field, approaching the phase instability state, the time of reorientation became less than $0.1 \mathrm{~s}$. The filament lamp has been used as a light source in this case. It illuminated all the film surface with the intensity below $1 \mathrm{~W} / \mathrm{cm}^{2}$. Therefore the DS reconstruction took place all over the film. 
2.2 Polarization, amplitude and spectral dependences.

Polarization diagram (Fig.3) shows the dependence of the inverse time of remagnetization vs azimuthal angle of the light polarization. Illumination by the light with $\mathbf{E} \mid[001]$ or $\mathbf{E} \mid[010]$ did not realize any changes of the DS. The velocity

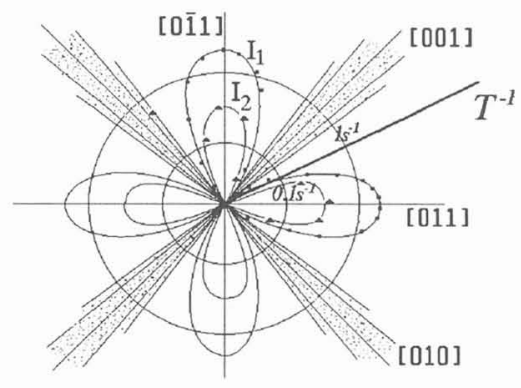
of the DS reconstruction in proportion depends upon the light intensity [4]. Its typical value was changed from $0.1 \mathrm{~s}$ to $10 \mathrm{~s}$. The threshold of polarized light intensity, initiated the PME (displacement of the domain walls) is less then $0,1 \mathrm{~W} / \mathrm{cm}^{2}$ and it depends upon the film coercivity and local magnetization of the neighbouring areas of the film. Besides that, variation of the light intensity change in proportion value of remanent magnetization (Fig. 4) in the same way as it changed the time of the DS reconstruction.

The external magnetic field, less than threshold $(0.7 \mathrm{Oe})$, applied in the film plane parallel to easy anisotropy axes did not destroy the effect. It only had to decrease or increase the reorientational time while its direction was parallel or perpendicular to magnetization of the increasing domains (Fig.4).

Figure 3. Polarization diagram of the effect (in polar coordinate). $\mathrm{Il}=10 \mathrm{~W} / \mathrm{cm}^{2}, \mathrm{I} 2=0.2 \mathrm{~W} / \mathrm{cm}^{2}$.

Measurement of the spectral dependence of photoinduced magnetization has been carried out using of

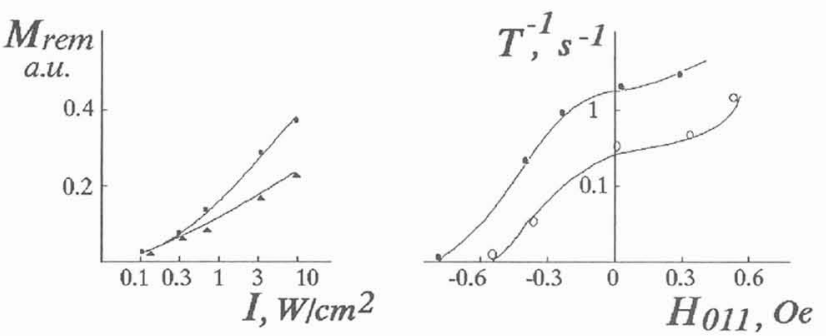

Figure 4. Light induced magnetization of garnet film vs light intensity measured: at once after switching off the light;

(the result of magnetic after-effect).

Figure 5. Velocity of the effect vs external magnetic field. the spectral filters. Illumination by the linearly polarized light $(I=0.05-1.0$ $\mathrm{W} / \mathrm{cm}^{2}$ ), of the correspondent colour in the visible wave band

magnetized the local area in the direction, obliged by orientation of polarization vector. Photomagnetic sensibility, (solid curve on Fig.6, dashed - absorption) determined as inverse time of remagnetization is decreased in a red edge of the spectral wave band, as well as in a narrow part of a green wave band. Such results are similar to those one, obtained for spectral dependence of permeability in garnet film at cryogen temperature and differ from the results obtained for bulk YIG:Si.

\subsection{Phase diagram.}

The effect is intensified by cooling of the film below the room temperature to about $200 \mathrm{~K}$ (the intensity of remanent magnetization is increased and the reorientation time is decreased in approximately 3 times). Further decreasing of the temperature induced a strong uniaxial anisotropy with the axis perpendicular to the film plane. That is destroyed the effect because of the spin-reorientational transitions in the [001] direction. Rising up the temperature as well as the thermal influence of high-intensive light decreased the effect dependence on polarization, increased the thermal magnetization and the magnetic after-effect.

In order to study the influence of heat treatment on parameters and photomagnetic properties of the films they were subjected to thermal annealing in atmosphere at temperatures $700-900 \mathrm{C}$. One of the most interesting result was a formation of the additional surface sublayer with the perpendicular anisotropy. Such a two-layered film lost the photomagnetic properties because of the mutual influence of the layers. However this allows us to widen the band of the spatial wavelength of magnetic records, copying by such film.

One more influential factor has been investigated in the experiment on mechanical pressure in the (100)-plane of the film. Applying along the easy anisotropy axes it has the similar effect on the DS such as polarized light either magnetic field. The film was also being magnetized in the direction, determined by orientation of the strain. 


\section{DISCUSSION}

Some conclusions about the ongin of the room-PME can be inferred from the obtained dependences. One of the 1

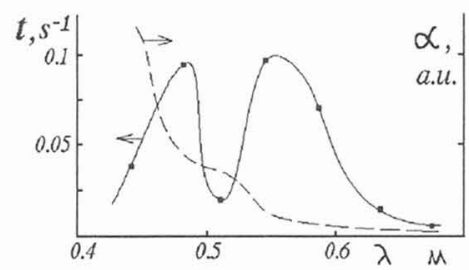

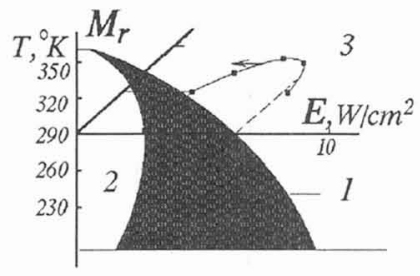

Figure 6. Spectral dependence of the effect \& absorption.

Figure 7. Phase diagram of the effect: 1-area of polarization-dependent effect. peculiarities, in comparison with the cryogen PME, is the reconstruction of the DS by means of the domain walls displacement and arising of the new phase of magnetization only on the domain walls. At low temperatures in bulks we also observed photoinduced rotation of magnetization. For all that cryogen photoinduced remagnetization could take place all over the illuminated sample. The obvious reason of this is more preferable energy conditions on the domain wall, where magnetic

moments must be rotated on the lesser angle than somewhere else. Another characteristic feature is a strong thermal disordering that did not have visible influence on the bulk DS at $77 \mathrm{~K}$. The observed after-effect of partial demagnetization (Fig.2f and Fig.4) is connected with such a thermal disordering.

The absence of the effect in bulk samples at $T>150 \mathrm{~K}$ has been explained by thermal activations of the "frozen" states of the orientally inquivalent Fe2+-ions. We suppose that photoinduced magnetization of the film under the light illumination at room temperature became a consequence of the strong magnetostriction in the (100)-plane. This magnetostriction is arisen from the difference of the film and substrate constants. It results in a corresponding mechanical strain that can approach magnetic system to phase instability state. The first-ordered importance of magnetostriction mechanism in displaying of the room-PME is confirmed, in particular, by the absence of this effect in other similar films which differ only by the lower magnetostriction constants. The observed difference in reconstruction of the DS at $300 \mathrm{~K}$ and at $77 \mathrm{~K}$ can be explained in the following way. At low temperatures photoinduced anisotropy reoriented magnetic moment in the direction of the easy anisotropy axis, determined by orientation of polarization vector. At room temperatures in film with a high magnetostriction the photoinduced strain under illumination is occurred resembling as in YIG:Si at $77 \mathrm{~K}$ [5]. Due to extending of the film, (if polarization vector $\mathbf{E}$ parallel to [011] ) or compression (if $\mathbf{E}$ perpendicular to [0-10]), the magnetization vector was reoriented in the opposite direction in accordance with Fig. 2.

The micromagnetic mechanism of the PME is usually explained by a model of photosensitive magnetoanisotropic centres. Such photoactive centres are based on $\mathrm{Fe} 2+$ ions, that can change their orientally inquivalent sites under illumination. Assuming of the $\mathrm{Fe} 2+$ ions presence in the crystal lattice is correct due to existing of non-controlled admixtures of $\mathrm{Pb}$ in $\mathrm{PbO}$-based flux, used in the process of epitaxy. Illumination of the film by polarized light cause a dependent on polarization electric recharging of such impurity centres and defects of the crystal lattice. In consequence, the distinguished anisotropy axis is arisen in the crystal. This phenomenon is accompanied by the photoinduced strain of the crystal lattice, that influence the DS of the film much stronger than it was in the bulk samples. Hence the value and direction of magnetization become polarization- dependent in consequence of the lattice distortion under illumination.

There are some uncertainties in this micromagnetic models connected with a problem of localized electronic states. So some authors developed the phenomenological description of the PME, that also can be enlarged on roomtemperature case. Taking into account measured characteristics of the investigated films one can estimate their optimum parameters (saturation magnetization, coercivity, anisotropy, magnetostriction and photomagnetic sensibility) analysing of the general energy balance equations. In particular, the room-PME can occur in finite range of coercivities ( $0.5-2 \mathrm{Oc}$ at estimated cations level 0.01 per formula). So the possible way of increasing the effect to enough for practical purposes is to select the films with a suitable concentration of the dopants.

Investigations of the room-PME are attractive not only for studying of photomagnetic spin-reorientational transitions, but also for application. As the films with a low energy of remagnetization are used for a high-sensitive analysis of magnetic field, so they can be utilized for copying and vizualizing low-frequency information, recorded on magnetic tapes. A spatially oriented high-sensitive magnetic field probe can be realized due to polarization sensibility of magnetization, that can be reoriented in the necessary direction by variation of polarization. Illumination by polarized light change the sensibility of magnetic probe and a threshold of its operation. Further techuological optimization of the films parameters can extend application of the PME.

\section{References}

[1]. R.Alben, E.M.Gyorgy, J.F.Dillon, J.P.Remeika, Phys.Rev. B5 (1972) 2560-2577.

[2]. K.Hisatake, T.Matsuyama, Japan J.Appl.Phys., 13 (1974) 2061 -2062.

[3]. A.B.Chizhik, A.Maziewski, A.Stupakiewicz, 14 Int. Col. on Magn. Films, Dusseldorf, 29 Aug-2 Sept. 1994, p. 786.

[4]. Sohatsky V., Kovalenko V. JETP Lett., 61 (1995) 1021-1025.

[5]. J.F.Dillon, E.M.Gyorgy, J.P.Remeika, Appl.Phys. Lett. 15 (1969) 221. 